TRANSACTIONS OF THE

AMERICAN MATHEMATICAL SOCIETY

Volume 355, Number 2, Pages 655-663

S 0002-9947(02)03135- 5

Article electronically published on September 6, 2002

\title{
A GENERALIZED MINKOWSKI PROBLEM WITH DIRICHLET BOUNDARY CONDITION
}

\author{
OLIVER C. SCHNÜRER
}

\begin{abstract}
We prove the existence of hypersurfaces with prescribed boundary whose Weingarten curvature equals a given function that depends on the normal of the hypersurface.
\end{abstract}

\section{INTRODUCTION}

Let $\tilde{M} \in \mathbb{R}^{n+1}$ be a strictly convex smooth hypersurface and $0<f \in C^{2, \alpha}\left(S^{n}\right)$. We denote by $\tilde{M}_{+}$an open subset of $\tilde{M}$ with smooth boundary $\partial \tilde{M}_{+}$(with respect to $\tilde{M}$ ) and $\tilde{M}_{+} \subsetneq \tilde{M}$. Let $F$ be a curvature function, as, for example $S_{n}$, the Gauß curvature, or $S_{k} \cdot S_{n}, 1 \leq k \leq n$, where $S_{k}$ denotes the $k$-th elementary symmetric polynomial

$$
S_{k}\left(\kappa_{i}\right)=\sum_{1 \leq i_{1}<\ldots<i_{k} \leq n} \kappa_{i_{1}} \cdot \ldots \cdot \kappa_{i_{k}} .
$$

A description of the curvature functions considered in this paper is given in Section 2 We denote the principal curvatures of a hypersurface $M$ by $\kappa_{i}(M), 1 \leq i \leq n$, and the outer unit normal of $\tilde{M}$ by $\nu_{\widetilde{M}}$. Under these assumptions we prove the following theorem.

Theorem 1.1. If $F\left(\kappa_{i}(\tilde{M})\right) \geq f\left(\nu_{\tilde{M}}\right)$ in $\tilde{M}_{+}$, then there exists a $C^{4, \alpha}$-hypersurface $M$ with

$$
F\left(\kappa_{i}(M)\right)=f(\nu)
$$

and $\partial M=\partial \tilde{M}_{+}$, where $\nu$ denotes an appropriately chosen unit normal of $M$.

More precisely, we look for a strictly convex hypersurface contained in the convex body determined by $\tilde{M}$, whose unit normal points inside the bounded component of $\mathbb{R}^{n+1} \backslash \overline{\left(\tilde{M}_{+} \cup M\right)}$ and $M \cup\left(\tilde{M} \backslash \tilde{M}_{+}\right)$is the boundary of a strictly convex body.

The paper is organized as follows. In Section 2 we introduce the curvature functions we are interested in, and in Section 3 we state our notation for differential geometry. Simple a priori estimates are proved in Section 4 and more complicated ones are proved in Section [5] Finally, we sketch in Section 6 how these a priori estimates can be used for the existence proof.

The classical Minkowski problem has been solved for smooth functions in any dimension in [3]. A generalization for other curvature functions has been obtained

Received by the editors November 8, 2000.

2000 Mathematics Subject Classification. Primary 35J65; Secondary 53C42.

Key words and phrases. Minkowski problem, Dirichlet problem, prescribed curvature, convex hypersurfaces.

(C)2002 American Mathematical Society 
recently in [5]. There are many papers that consider a Dirichlet problem for equations of prescribed curvature. The most useful ones for our estimates were [12] and [6]. We remark that our allowing of the dependence of $f$ on $\nu$ without imposing structure conditions on $f$ besides positivity and smoothness seems to be new.

We wish to thank Jürgen Jost and the Max Planck Institute for Mathematics in the Sciences, Leipzig, where this research was carried out, for their hospitality. We would also like to thank Claus Gerhardt, Heidelberg, who brought the Minkowski problem to our attention.

\section{Curvature Functions}

In this paper, we consider symmetric curvature functions defined in the positive cone $\Gamma_{+} \subset \mathbb{R}^{n}$ which are of the class $C^{2, \alpha}\left(\Gamma_{+}\right) \cap C^{0}\left(\overline{\Gamma_{+}}\right)$, positive homogeneous and satisfy

$$
\begin{gathered}
F_{i}=\frac{\partial F}{\partial \kappa_{i}}>0 \text { in } \Gamma_{+}, \\
\left.F\right|_{\partial \Gamma_{+}}=0 .
\end{gathered}
$$

For a positive definite symmetric matrix $\left(h_{i j}\right) \in \operatorname{Sym}^{+}(n, \mathbb{R})$ with eigenvalues $\kappa_{i}$, $1 \leq i \leq n$, we define

$$
F\left(h_{i j}\right):=F\left(\kappa_{i}\right),
$$

where the ambiguous notation should not cause any difficulties. Given $F \in$ $C^{2, \alpha}\left(\mathrm{Sym}^{+}\right) \cap C^{0}\left(\overline{\mathrm{Sym}^{+}}\right)$, we can define

$$
F^{i j}=\frac{\partial F}{\partial h_{i j}}, \quad F^{i j, k l}=\frac{\partial^{2} F}{\partial h_{i j} \partial h_{k l}},
$$

so that, for an appropriate interpretation of the right-hand side,

$$
F^{i j} \xi_{i} \xi_{j}=\frac{\partial F}{\partial \kappa_{i}}\left|\xi_{i}\right|^{2} \quad \forall \xi \in \mathbb{R}^{n}
$$

and $F^{i j}$ is diagonal if $h_{i j}$ is diagonal.

We define the elementary symmetric polynomials by

$$
S_{k}(\kappa):=\sum_{1 \leq i_{1}<\ldots<i_{k} \leq n} \kappa_{i_{1}} \cdot \ldots \cdot \kappa_{i_{k}}, \quad 1 \leq k \leq n,
$$

and furthermore,

$$
\begin{aligned}
\tilde{S}_{k}(\kappa) & :=\frac{1}{S_{k}\left(\frac{1}{\kappa_{i}}\right)}=\frac{S_{n}(\kappa)}{S_{n-k}(\kappa)}, \\
\sigma_{k} & :=\left(S_{k}\right)^{1 / k}, \quad \tilde{\sigma}_{k}:=\left(\tilde{S}_{k}\right)^{1 / k}, \\
\gamma_{k} & :=\left(\sum_{|\alpha|=k} \kappa^{\alpha}\right)^{1 / k}, \quad \tilde{\gamma}_{k}(\kappa):=\frac{1}{\gamma_{k}\left(\frac{1}{\kappa_{i}}\right)},
\end{aligned}
$$

where $\alpha$ is a multi-index. 
In this paper we will consider curvature functions $F$ of the form

$$
F=\sigma^{\alpha} \cdot \gamma^{\alpha^{\prime}}, \quad \text { where } \sigma^{\alpha} \equiv \prod_{k=1}^{n} \sigma_{k}^{\alpha_{k}}
$$

and we assume $\alpha_{n}>0$ and $\alpha, \alpha^{\prime} \geq 0$ in the sense of multi-indices. These functions obviously fulfill the conditions for curvature functions from the beginning of this section, and, furthermore,

$$
\tilde{F}\left(\kappa_{i}\right):=\frac{1}{F\left(\frac{1}{\kappa_{i}}\right)}=\tilde{\sigma}^{\alpha} \cdot \tilde{\gamma}^{\alpha^{\prime}} .
$$

Defining an abstract class of curvature functions for which the existence proof can be carried out seems to require many restrictions; so we mention only these examples. The fact that $F$ contains a positive power of the Gauß curvature is restrictive, but we have to ensure $\left.F\right|_{\partial \Gamma_{+}}=0$ to preserve the convexity of the hypersurfaces appearing in our existence proof as well as a kind of concavity of $F$ to deduce $C^{2, \alpha}$ estimates from the $C^{2}$ a priori estimates.

Lemma 2.1. For $F$ as above, the function

$$
\hat{\tilde{F}}=\log \tilde{F}=-\log F\left(\frac{1}{\kappa_{i}}\right)
$$

is concave and the following properties are equivalent provided $0<c \leq F \leq \frac{1}{c}<\infty$ :

$$
\begin{aligned}
\kappa_{1} & \rightarrow 0, \\
\kappa_{n} & \rightarrow+\infty, \\
\operatorname{tr} F^{i j}=F^{i j} \delta_{i j} & \rightarrow+\infty,
\end{aligned}
$$

when $0<\kappa_{1} \leq \ldots \leq \kappa_{n}$.

Proof. The concavity follows from the properties of curvature functions collected in [4.

$\kappa_{1} \rightarrow 0$ and $\left.F\right|_{\partial \Gamma_{+}}=0$ force $\kappa_{n} \rightarrow \infty . \kappa_{1} \geq c>0$ and $\kappa_{n} \rightarrow \infty$ contradict $F \leq \frac{1}{c}$. Here and below we use $c$ to denote a positive constant that may change its value if necessary. $\operatorname{tr} F^{i j} \rightarrow \infty$ is impossible for $\kappa$ in a compact subset of $\Gamma_{+}$, and so $\kappa_{1} \rightarrow 0$ or $\kappa_{n} \rightarrow \infty$.

Let $F=\sigma^{\alpha} \cdot \gamma^{\alpha^{\prime}}$ as above. Then

$$
\operatorname{tr} F^{i j} \geq \frac{\alpha_{n}}{n} \cdot \sigma^{\alpha} \cdot \frac{1}{\kappa_{1}} \cdot \gamma^{\alpha^{\prime}} \geq \frac{\alpha_{n}}{n} \cdot c \cdot \frac{1}{\kappa_{1}} .
$$

Therefore, $\kappa_{1} \rightarrow 0$ implies tr $F^{i j} \rightarrow+\infty$.

We may assume that $F$ is positive homogeneous of degree one.

When we consider a hypersurface $M$ with induced metric $g_{i j}$, we compute the eigenvalues of $h_{i j}$ with respect to $g_{i j}$, or equivalently we compute the eigenvalues of $h_{i k} g^{k j} \equiv h_{i}^{j},\left(g^{i j}\right)=\left(g_{i j}\right)^{-1}$. Then the chain rule yields [10]

$$
\frac{\partial F}{\partial g_{i j}}=-F^{i k} h_{k}^{j}
$$




\section{Notation}

In Euclidean coordinate systems the induced metric $g_{i j}$ and the inverse $g^{i j}$ of the induced metric of graph $u$ are given by

$$
\begin{aligned}
g_{i j} & =\delta_{i j}+u_{i} u_{j}, \\
g^{i j} & =\delta^{i j}-\frac{u^{i} u^{j}}{v^{2}}, \\
u^{i} & =u_{j} \delta^{i j}, \\
v & =\sqrt{1+|D u|^{2}}=\sqrt{1+u_{i} u^{i}},
\end{aligned}
$$

where Latin indices range from 1 to $n$ and refer to quantities of graph $u$, except for $r, s$ and $t$, which only range to $n-1$ and refer to an $(n-1)$-dimensional boundary. The Einstein summation convention is always used. Greek indices range from 0 to $n$ and refer to $\mathbb{R}^{n+1}$.

Let $x^{\alpha}$ be a Euclidean coordinate system for $\mathbb{R}^{n+1}, \nu^{\alpha}$ the "upwards pointing" unit normal of graph $u, \Gamma_{\text {.. }}$ the induced Christoffel symbols and $h_{i j}$ the second fundamental form. We will choose coordinates such that $h_{i j}$ is positive definite for a strictly convex hypersurface. These quantities are related by the Gauß formula $\left(x_{; i j}^{\alpha}\right.$ indicate covariant derivatives, while $u_{i j}$ and $x_{, i j}^{\alpha}$ denote partial derivatives):

$$
\begin{aligned}
x_{; i j}^{\alpha} & =-h_{i j} \nu^{\alpha}, \\
\nu^{\alpha} & =\frac{1}{v}\left(-u^{i}, 1\right), \\
x_{; i j}^{\alpha} & =x_{, i j}^{\alpha}-\Gamma_{i j}^{k} x_{k}^{\alpha}, \\
\Gamma_{i j}^{k} & =\frac{1}{v^{2}} u^{k} u_{i j}, \\
h_{i j} & =-\left(u_{i j}-\Gamma_{i j}^{k} u_{k}\right) \cdot v=-\frac{u_{i j}}{v} .
\end{aligned}
$$

As $M$ is strictly convex, $M \ni z \mapsto \nu(z)$ is a diffeomorphism from $M$ to $\nu(M) \subset$ $S^{n}$. For $x \in \nu(M)$ we define the supporting function $u$ (the ambiguous use of $u$ should not cause any difficulties) by

$$
u(x)=x^{\alpha} \delta_{\alpha \beta}\left(\nu^{-1}\right)^{\beta}(x)=\left\langle x, \nu^{-1}(x)\right\rangle,
$$

i.e., the scalar product is the usual scalar product in $\mathbb{R}^{n+1}$. We remark that the original hypersurface can be recovered from the support function [3]. The eigenvalues of

$$
\left(u_{; i j}+u \sigma_{i j}\right)(x), \quad x \in S^{n},
$$

with respect to the standard metric $\sigma_{i j}$ of $S^{n}$ are the inverses of the principal curvatures of $M$ at $\nu^{-1}(x)$, where $u_{; i j}$ denotes covariant derivatives with respect to the metric $\sigma_{i j}$. For covariant derivatives on $S^{n}$ we have

$$
u_{; i j k l}=u_{; k l i j}+2 u_{; i j} \sigma_{k l}-2 u_{; k l} \sigma_{i j}+u_{; k j} \sigma_{i l}-u_{; i l} \sigma_{k j}
$$

\section{Preliminary a Priori Estimates}

We assume that $M$ is a prospective solution and prove a priori estimates for this solution. Later on, we will deform our problem and use these estimates. Therefore, 
we will have to ensure especially that $M$ lies inside the convex body determined by $\tilde{M}$. This can be achieved if we approximate $f$ so that $\tilde{M}$ is a strict supersolution,

$$
F\left(\kappa_{i}(\tilde{M})\right)>f\left(\nu_{\tilde{M}}\right) .
$$

In view of our a priori estimates, we may afterwards choose a subsequence of solutions of the modified problems that converge to a solution of the original problem.

4.1. $C^{0}$ - and $C^{1}$-estimates. It is possible to represent $M$ as a graph over an appropriately chosen part of a small sphere with center in $\tilde{M} \backslash \overline{\tilde{M}_{+}}$, graph $\left.u\right|_{\Omega}=M$, such that $|u|_{1}$ is bounded. The $C^{0}$ bounds follow from the geometric setting and the convexity of $M$; the $C^{1}$ bounds follow as the angle between each half-line starting from the center of the small sphere and $M$ in an intersection point is a priori bounded from below. In our setting, this follows by simple geometric reasoning. A proof for a much more general situation can be found in 9 .

4.2. A special coordinate system. In view of the uniform $C^{1}$-estimates, we may rotate our original Euclidean coordinate system appropriately so that we have the following situation: An arbitrary but fixed point $x_{0}$ of $\partial \tilde{M}_{+}$is the origin of our coordinate system. Furthermore, we have a function $\omega: \hat{\hat{B}}_{r} \rightarrow \mathbb{R}, \hat{\hat{B}}_{r}:=\left\{x^{\prime \prime} \in\right.$ $\left.\mathbb{R}^{n-1}:\left|x^{\prime \prime}\right|<r\right\}, \omega(0)=0, D \omega(0)=0$, such that $M \cap\left(\hat{B}_{r} \times \mathbb{R}\right)=\left.\operatorname{graph} u\right|_{\Omega}$, where $\hat{B}_{r}:=\left\{x^{\prime} \in \mathbb{R}^{n}:\left|x^{\prime}\right|<r\right\}, \Omega:=\hat{B}_{r} \cap\left\{\left(x^{\prime}, x^{n}\right): x^{n}>\omega\left(x^{\prime}\right)\right\}$ and $u$ is an appropriate function whose graph locally coincides with $M . r>0$ is uniformly bounded from below by a positive constant which is, in particular, independent of $x_{0}$ and the second fundamental form of $M . \omega$ and its derivatives are a priori bounded as well as $|u|_{1}$. The function $u$ is as smooth as $M$, but its derivatives of order greater than one are not yet a priori bounded. In the same way in which $M$ is locally represented as graph $u$, we may assume that $\tilde{M}=\left.\operatorname{graph} \tilde{u}\right|_{\Omega}$, but the derivatives of $\tilde{u}$ are a priori bounded. Furthermore, we may assume $u(0)=\tilde{u}(0)=0$ and $u_{r}(0)=\tilde{u}_{r}(0)=0,1 \leq r \leq n-1$.

4.3. Tangential $C^{2}$-estimates at the boundary. We choose a coordinate system as described above. On $\partial \Omega, u$ and $\tilde{u}$ coincide; so we may differentiate

$$
(\tilde{u}-u)\left(x^{\prime \prime}, \omega\left(x^{\prime \prime}\right)\right)=0
$$

twice and obtain (because $D \omega(0)=0$ for $1 \leq r, s \leq n-1$ at the origin)

$$
u_{r s}=\tilde{u}_{r s}+(\tilde{u}-u)_{n} \omega_{r s} .
$$

All terms on the right-hand side are bounded. So $\left|u_{r s}\right|$, i.e., the tangential derivatives of $u$, are a priori bounded.

\section{A Priori Estimates}

Compare this proof to the respective ones in [1, [2], [7], [6], [12] and [10].

5.1. Mixed $C^{2}$-estimates at the boundary. We consider the situation for a fixed point of $\partial M$ in a coordinate system as described in Section 4.2

We differentiate the equation

$$
F\left(h_{i j}, g_{i j}\right)=f\left(\nu^{\alpha}\right) \equiv f\left(u_{i}\right)
$$


with respect to $x^{k}$ and use

$$
h_{i j, k}=-\frac{1}{v} u_{i j k}+\frac{1}{v^{3}} u^{l} u_{l k} u_{i j} ;
$$

thus,

$$
f_{p_{i}} u_{i k}=-\frac{1}{v} F^{i j} u_{i j k}-\frac{1}{v^{2}} F u^{l} u_{l k}-F^{i l} h_{l}^{j}\left(u_{i k} u_{j}+u_{i} u_{j k}\right) .
$$

Therefore we define the linear operator $L$ by

$$
L w:=\frac{1}{v} F^{i j} w_{i j}+\frac{1}{v^{2}} F u^{l} w_{l}+F^{i l} h_{l}^{j}\left(u_{j} w_{i}+u_{i} w_{j}\right)+f_{p_{i}} w_{i},
$$

and for $t<n$ we define

$$
T:=\frac{\partial}{\partial x^{t}}+B_{t r} x^{r} \frac{\partial}{\partial x^{n}}-B_{t}^{r} x_{n} \frac{\partial}{\partial x^{r}},
$$

where

$$
\omega\left(x^{\prime}\right)=\frac{1}{2} B_{r s} x^{r} x^{s}+O\left(\left|x^{\prime}\right|^{3}\right), \quad x^{\prime}=\left(x^{1}, \ldots, x^{n-1}\right),
$$

$B_{t}^{r} \equiv B_{t s} \delta^{r s}, x_{n}=x^{i} \delta_{i n}$ and $r, s$ and $t$ run from 1 to $n-1$ as usual.

From the definition of $L$ and homogeneity, it is easy to see that

$$
|L T(u-\tilde{u})| \leq c \cdot\left(1+\operatorname{tr} F^{i j}\right),
$$

where, here and in the following, $c$ is bounded from above by estimated quantities.

Furthermore, in view of the $C^{1}$-estimates we have

$$
|T(u-\tilde{u})| \leq c \quad \text { in } \bar{\Omega},
$$

and, due to the definition of $T$,

$$
|T(u-\tilde{u})| \leq c \cdot|x|^{2} \quad \text { on } \partial \Omega .
$$

We consider the function

$$
\vartheta:=(\tilde{u}-u)+\alpha d-\mu d^{2}
$$

in a domain $\Omega_{\delta}:=\Omega \cap B_{\delta}(0)$, where $d$ is the distance function from $\partial \Omega$ in $\mathbb{R}^{n}$, and show that it satisfies a nice differential equation for $\alpha, \delta>0$ small and $\mu$ large.

As graph $\tilde{u}$ is strictly convex, we fix $\varepsilon>0$ such that $-\tilde{u}_{i j} \geq \varepsilon \delta_{i j}$ in the matrix sense. We compute

$$
L \vartheta \leq-\frac{\varepsilon}{v} \operatorname{tr} F^{i j}-2 \mu \frac{1}{v} F^{i j} d_{i} d_{j}+c+c \cdot(\alpha+\delta \mu) \cdot\left(1+\operatorname{tr} F^{i j}\right) .
$$

We use $-F^{r s} d_{r} d_{s} \leq 0,1 \leq r, s \leq n-1$, as well as $\left|F^{n r}\right| \leq \frac{1}{2} \operatorname{tr} F^{i j}$ and $d_{i} \approx \delta_{i n}$; so

$$
L \vartheta \leq-\frac{\varepsilon}{v} \operatorname{tr} F^{i j}-\mu \frac{1}{v} F^{n n}+c+c \cdot(\alpha+\delta \mu) \cdot\left(1+\operatorname{tr} F^{i j}\right) .
$$

From Lemma 2.1, we infer that for sufficiently large fixed $\mu$ the constant $c$ can be absorbed by the first two terms on the right-hand side. For small $\alpha, \delta>0$ we obtain

$$
L \vartheta \leq-\frac{1}{3} \frac{\varepsilon}{v} \operatorname{tr} F^{i j} .
$$

Furthermore, when $\delta>0$ is small,

$$
\vartheta \geq 0 \quad \text { on } \partial \Omega_{\delta}
$$


We consider the function

$$
\Theta:=A \vartheta+B|x|^{2} \pm T(u-\tilde{u}) .
$$

We fix $B \gg 1$, get $\Theta \geq 0$ on $\partial \Omega_{\delta}$, and deduce from Lemma 2.1 that $\operatorname{tr} F^{i j} \geq \frac{1}{c}>0$; so $L \Theta \leq 0$ for $A \gg B$. The maximum principle yields $\Theta \geq 0$ in $\Omega_{\delta}$. As $\Theta(0)=0$, we have $\Theta_{n}(0) \geq 0$, which in turn immediately gives $\left|u_{t n}(0)\right| \leq c, 1 \leq t \leq n-1$.

5.2. Normal $C^{2}$-estimates at the boundary. We may assume that the infimum of the invariantly defined function

$$
\partial \Omega \ni x \mapsto \inf _{0 \neq \zeta \in T_{x} \partial \Omega} \frac{h_{i j} \zeta^{i} \zeta^{j}(x)}{g_{i j} \zeta^{i} \zeta^{j}(x)}
$$

equals $h_{11}\left(x_{0}\right) / g_{11}\left(x_{0}\right)$, and fix a coordinate system around $x_{0}$ as in Section 4.2 We choose smooth vector fields $\xi_{i}, 1 \leq i \leq n$, around $x_{0}$ such that $\xi_{n}$ equals the inner unit normal to $\partial \Omega, \xi_{1}\left(x_{0}\right)=e_{1}\left(x_{0}\right)$, and the vectors $\xi_{i}$ are pointwise orthonormal with respect to the Euclidean metric.

From $\tilde{u}-u=0$ on $\partial \Omega$, we deduce along $\partial \Omega$ the well-known relation [12]

$$
\begin{aligned}
0 & =\xi_{r}^{i} \xi_{s}^{j}(\tilde{u}-u)_{i j}+\xi_{n}^{i}(\tilde{u}-u)_{i}\left(-\xi_{r}^{l} \xi_{s}^{j} \delta_{j k}\left(\xi_{n}^{k}\right)_{l}\right) \\
& \equiv \nabla_{r s}(\tilde{u}-u)+(\tilde{u}-u)_{\nu} C_{r s} .
\end{aligned}
$$

We wish to estimate $-\nabla_{11} u\left(x_{0}\right)$ from below by a positive constant. If $-\nabla_{11} u\left(x_{0}\right) \geq$ $-\frac{1}{2} \nabla_{11} \tilde{u}\left(x_{0}\right)$, we have such an estimate; otherwise we deduce from (5.17) that $C_{11}$ is locally bounded from below by a positive constant. As $\left|\nabla_{11} u\right|$ is already bounded on $\partial \Omega$, this means that

$$
\partial \Omega \ni x \mapsto-\nabla_{11} u+a|x|^{2}
$$

attains its infimum in $x_{1}$ near $x_{0}=0$ for $a \gg 1$, and thus

$$
c\left(x_{1}\right) \equiv-\nabla_{11} u\left(x_{1}\right)+a\left|x_{1}\right|^{2} \leq-\nabla_{11} u(x)+a|x|^{2}, \quad x \in \partial \Omega,
$$

or, in view of (5.17), for $x \in \partial \Omega$,

$$
\begin{aligned}
u_{\nu}(x) & \geq C_{11}^{-1}(x) \cdot\left(\nabla_{11} \tilde{u}(x)+c\left(x_{1}\right)-a|x|^{2}\right)+\tilde{u}_{\nu}(x) \\
& \equiv \gamma \in C^{2}, \quad|\gamma|_{2} \leq c .
\end{aligned}
$$

For $\Theta:=A \vartheta+B\left|x-x_{1}\right|^{2}-\gamma(x)+u_{\nu}(x)$, when $\gamma$ is extended appropriately, we deduce as in Section 5.1 that $-\nabla_{n n} u\left(x_{1}\right)$ is bounded from above. Lemma [2.1 implies a positive lower bound for $-\nabla_{11} u\left(x_{1}\right)$, thus also for $-\nabla_{11} u\left(x_{0}\right), h_{11}\left(x_{0}\right) / g_{11}\left(x_{0}\right)$ and $h_{\zeta \zeta} / g_{\zeta \zeta}, \zeta \in T \partial \Omega$. In view of Lemma 1.2 in [2] (or Young's inequality) and Lemma 2.1, we deduce a bound for the second derivatives of $u$ on $\partial \Omega$, and $0<\frac{1}{c} \leq \kappa_{i} \leq c$ for all eigenvalues $\kappa_{i}$ of $h_{i k} g^{k j}$.

5.3. Interior $C^{2}$-estimates. We proceed as in [5] and transfer the situation to $S^{n}$ via the Gauß map $M \ni x \mapsto \nu(x) \in S^{n}$.

For the estimates here, indices denote covariant derivatives with respect to $\sigma_{i j}$, $u$ is the support function of $M$, and we remark that

$$
\begin{aligned}
F\left(h_{i k} g^{k j}\right) & =\left(\tilde{F}\left(\left(u_{i k}+u \sigma_{i k}\right) \sigma^{k j}\right)\right)^{-1}, \\
\tilde{F}\left(\left(u_{i k}+u \sigma_{i k}\right)(x)\right. & =(f(x))^{-1}, \quad x \in \nu(M) \subset S^{n} .
\end{aligned}
$$


On $\partial M$ the eigenvalues of the second fundamental form are a priori bounded from below and from above. Assume that

$$
\frac{1}{\kappa_{1}(M)}+\ldots+\frac{1}{\kappa_{n}(M)}=\sigma^{i j}\left(u_{i j}+u \sigma_{i j}\right) \equiv \sigma^{i j} w_{i j}
$$

attains its maximum at an interior point $\nu^{-1}\left(x_{0}\right)$ of $M$. So we have there

$$
\begin{aligned}
0 & \geq\left(\sigma^{i j} w_{i j}\right)_{k l} \\
& =\sigma^{i j}\left(u_{i j k l}+u_{k l} \sigma_{i j}\right) \\
& =\sigma^{i j}\left(u_{k l i j}+2 u_{i j} \sigma_{k l}-u_{k l} \sigma_{i j}+u_{k j} \sigma_{i l}-u_{i l} \sigma_{k j}\right) \\
& =\Delta\left(u_{k l}\right)+2 \Delta u \sigma_{k l}-n u_{k l} \\
& =\Delta\left(w_{k l}\right)-n w_{k l}+\sigma^{i j} w_{i j} \sigma_{k l},
\end{aligned}
$$

where $\Delta(\cdot)=\sigma^{i j}(\cdot)_{i j}$. Define

$$
\hat{\tilde{F}}^{k l}=\frac{\partial \hat{\tilde{F}}}{\partial w_{k l}}\left(w_{i j}\right) .
$$

As $\hat{\tilde{F}}^{i j}$ is positive,

$$
\begin{aligned}
0 & \geq \hat{\tilde{F}}^{k l}\left(\sigma^{i j} w_{i j}\right)_{k l} \\
& =\hat{\tilde{F}}^{k l} \Delta\left(w_{k l}\right)-n \hat{\tilde{F}}^{k l} w_{k l}+\sigma^{i j} w_{i j} \hat{\tilde{F}}^{k l} \sigma_{k l} .
\end{aligned}
$$

$\tilde{F}$ is homogeneous of degree one, and so

$$
\hat{\tilde{F}}^{k l} w_{k l}=\frac{1}{\tilde{F}} \frac{\partial \tilde{F}}{\partial w_{k l}} w_{k l}=1 .
$$

We differentiate $\hat{\tilde{F}}\left(w_{i k} \sigma^{k j}\right)=-\log f(x)$ and use the concavity

$$
\sigma^{i j} \hat{\tilde{F}}^{k l} w_{k l ; i j} \geq-\Delta \log f(x),
$$

and so we obtain

$$
0 \geq-\Delta \log f-n+\left(\sigma^{i j} w_{i j}\right) \cdot \hat{\tilde{F}}^{k l} \sigma_{k l} .
$$

As $\hat{\tilde{F}}^{k l} \sigma_{k l}$ is bounded from below by a positive constant (show that this quantity tends to infinity when an eigenvalue of $w_{i j}$ approaches zero as in the proof of Lemma 2.1) and $f$ is a given function, we conclude that $\sigma^{i j} w_{i j}$ is a priori bounded, i.e., $\kappa_{i}(M), 1 \leq i \leq n$, is bounded from below by a positive constant, and in view of Lemma 2.1 also from above. This proves the a priori estimate for the eigenvalues of the second fundamental form and for the second derivatives of a function representing $M$ locally as a graph in coordinate systems as in Section 4.1 or 4.2

5.4. $C^{2, \alpha}$-estimates and further estimates. As mentioned in [4, the functions $\sigma_{k}$ and $\gamma_{k}, 1 \leq k \leq n$, are concave and monotone increasing. Our a priori estimates now guarantee that

$$
\log F=\sum_{k} \alpha_{k} \log \sigma_{k}+\sum_{k} \alpha_{k}^{\prime} \log \gamma_{k}
$$


is uniformly elliptic and strictly concave. So we can apply Krylov-Safanov theory, Chapter 14.13 in [11], and deduce $C^{2, \alpha}$ a priori estimates. Finally, we deduce $C^{4, \alpha}$ a priori estimates by using Schauder theory.

\section{EXISTENCE}

To prove the existence of the hypersurface $M$ that we are looking for, we may proceed as in [8] or 9 and deform our problem into a local problem such that $M$ and $\tilde{M}_{+}$are representable as graphs in a single Euclidean coordinate system. During this step we represent solutions of the deformed problem in a coordinate system as in Section 4.1. If this deformation is carried out appropriately, the degree mod 2 implies that it suffices to solve the local problem. The maximum principle yields that solutions of the deformed problem fulfill analogous geometric conditions as stated next to the main theorem. In view of the uniqueness of a prospective solution of the local problem and the a priori estimates, a standard continuity method yields the existence for the local problem. This in turn yields a solution of our original problem via degree mod 2 theory.

\section{REFERENCES}

1. L. Caffarelli, L. Nirenberg, J. Spruck: The Dirichlet Problem for Nonlinear Second-Order Elliptic Equations I. Monge-Ampère Equation, Comm. Pure Applied Math. 37 (1984), 369402. MR 87f:35096

2. L. Caffarelli, L. Nirenberg, J. Spruck: The Dirichlet problem for nonlinear second order elliptic equations, III: Functions of the eigenvalues of the Hessian, Acta Math. 155 (1985), 261-301. MR 87f:35098

3. S.-Y. Cheng, S.-T. Yau: On the Regularity of the Solution of the n-Dimensional Minkowski Problem, Comm. Pure Appl. Math. 29 (1976), 495-516. MR 54:11247

4. C. Gerhardt: Closed Weingarten hypersurfaces in Riemannian manifolds, J. Differential Geom. 43 (1996), 612-641. MR 97g:53067

5. B. Guan, P. Guan: Convex hypersurfaces of prescribed curvature, to appear in Ann. of Math..

6. B. Guan: The Dirichlet problem for Monge-Ampère equations in non-convex domains and spacelike hypersurfaces of constant Gauß curvature, Trans. Amer. Math. Soc. 350, No. 12, (1998), 4955-4971. MR 99b:53055

7. B. Guan, J. Spruck: Boundary-value problems on $\mathbb{S}^{n}$ for surfaces of constant Gauss curvature, Ann. Math. 138 (1993), 601-624. MR 94i:53039

8. N. M. Ivochkina, F. Tomi: Locally convex hypersurfaces of prescribed curvature and boundary, Calc. Var. and PDEs 7 (1998), 293-314. MR 2000b:53043

9. T. Nehring: Hypersurfaces of prescribed Gauß curvature and boundary in Riemannian manifolds, J. reine angew. Math. 501 (1998), 143-170. MR 99i:53041]

10. O. C. Schnürer: The Dirichlet problem for Weingarten hypersurfaces in Lorentz manifolds, Math. Z. 242 (2002), 159-181.

11. M. E. Taylor: Partial differential equations. III. Nonlinear equations, Applied Mathematical Sciences, 117, Springer, New York, 1997, xxii+608 pp. MR 98k:35001

12. N. S. Trudinger: On the Dirichlet problem for Hessian equations, Acta Math. 175 (1995), 151-164. MR 96m:35113

Max Planck Institute for Mathematics in the Sciences, Inselstrasse 22-26, D-04103 Leipzig, Germany

Current address: Department of Mathematics, Harvard University, One Oxford Street, Cambridge, MA 02138, USA

E-mail address: Oliver.Schnuerer@mis.mpg.de

E-mail address: schnuere@math.harvard.edu 